\title{
Inhibition of Poly(ADP-Ribose) Polymerase Increased Lipid Accumulation Through SREBP1 Modulation
}

\author{
Jing Pang ${ }^{\mathrm{a}}$ Ju Cui ${ }^{\mathrm{a}}$ Chao Xib Tao Shen ${ }^{\mathrm{a}}$ Huan Gong ${ }^{\mathrm{a}}$ Lin Dou \\ Yajun Lin ${ }^{\mathrm{a}}$ Tiemei Zhang ${ }^{\mathrm{a}}$ \\ aThe MOH Key Laboratory of Geriatrics, Beijing Hospital, National Center of Gerontology, Beijing, \\ ${ }^{b}$ College of Life Sciences, Beijing Normal University, Beijing, China
}

\section{Key Words}

Poly(ADP-ribose) polymerase $\cdot$ Lipid synthesis $\bullet$ High fat state $\cdot$ Metabolism

\begin{abstract}
Background/Aims: Excess energy intake leads to metabolic dysfunction, accompanied by oxidative stress and poly(ADP-ribose) polymerase (PARP) activation. Methods: To determine the role of PARP activation in the incidence of metabolic dysfunction, PJ34, the PARP inhibitor, was administered to the oleic acid-treated hepatoma cells and high-fat diet-fed mice. The expression of genes was detected by quantitative real-time PCR and western blotting. Lipid droplets in the cells and tissues were stained with Oil Red O. Results: PJ34 treatment aggravated oleic acid-induced lipid accumulation in hepatoma cells and induced SREBP1 expression by modulating the modification of transcription factor specificity protein 1 (Sp1). The high-fat diet-mice exhibited hyperglycemia, insulin resistance and lipid accumulation after 3 months of feeding. Although the serum level of lipid was not altered after PJ34 treatment, the expression level of lipogenic gene was up-regulated and the lipid accumulation was increased in the liver tissues of high-fat diet + PJ34-treated mice. In the high-fat diet + PJ34-treated mice, the insulin sensitivity was slightly changed and the levels of blood glucose and serum insulin were decreased compared with the mice fed with a high-fat diet alone. Conclusion: Taken together, PARP inhibition up-regulated the expression level of lipogenic gene and significantly induced lipid accumulation in the liver, which might worsen lipid metabolism disorders. These data will guide future research into the application of PARP inhibitors in the management of metabolic diseases.

(C) 2018 The Author(s)

Published by S. Karger AG, Basel
\end{abstract}

\section{Introduction}

Poly (ADP-ribose) polymerase (PARP) is a member of the enzyme family which is involved in DNA damage and repair. PARP can be activated by DNA strand breaks, irregular DNA structures or other post-translational modifications [1]. PARP activation can regulate the protein function, chromatin compaction and gene expression by modifying target 


\section{Cellular Physiology Cell Physiol Biochem 2018;49:645-652 \begin{tabular}{ll|l} 
and Biochemistry & $\begin{array}{l}\text { DOI: 10.1159/000493028 } \\
\text { Published online: } 30 \text { August, } 2018\end{array}$ & $\begin{array}{l}\text { @ } 2018 \text { The Author(s). Published by S. Karger AG, Basel } \\
\text { www.karger.com/cpb }\end{array}$ \\
\hline
\end{tabular} \\ Pang et al.: PARP Inhibition Increased Lipid Accumulation}

proteins via poly(ADP-ribosyl)ation [2]. However, over-activation of PARP can lead to cellular necrosis because of NAD and ATP depletion. PARP activation, which is a protective response to DNA damage, also plays a role in cellular dysfunction.

Excess energy intake can provoke the over-production of reactive oxygen species and impair the biological macromolecules, thus leading to metabolic dysfunction in patients with type 2 diabetes mellitus [3,4]. During the process of excess energy intake, PARP is activated by oxidative stress and associated with glucose metabolic disorders. PARP1 deletion increases the mitochondrial biosynthesis and prevents the insulin resistance induced by high-fat diet through increasing NAD+ content and SIRT1 activity in obese mice [5]. PARP inhibitor improves the insulin sensitivity in hepatocytes via NAD-SIRT1 modulation. Accordingly, PARP inhibition plays a positive role in glucose metabolism. Nevertheless, the effect of PARP inhibitors upon the lipid metabolism remains elusive.

PARP1 gene deletion has been reported to enhance the lipid accumulation in liver and exacerbate high fat-induced obesity [6]. The beneficial function of PARP may be impaired when the PARP1 gene is deleted. Therefore, partial inhibition of the activated PARP is worthy of being investigated with respect to metabolic dysfunction therapy. Whether PARP inhibitors exert identical effect on lipid metabolism with PARP1 gene deletion or not is urgently to be elucidated.

To evaluate the effect of PARP inhibitors upon lipid metabolism, oleic acid-treated hepatocytes were treated with PJ34 and the mouse models were fed with high-fat diet. The results have implications for the clinical application of PARP inhibitors in the management of metabolic diseases.

\section{Materials and Methods}

\section{Cell culture}

Mouse hepatoma HEP1-6 cells obtained from American Type Culture Collection, were cultured in DMEM (Invitrogen, Grand Island, NY) containing $25 \mathrm{mM}$ glucose supplemented with $10 \%$ fetal bovine serum (Biowest, France). Cells were grown at $37^{\circ} \mathrm{C}$ in a $5 \%$ CO2-humidified atmosphere. For high fat treatment, cells were treated with $500 \mu \mathrm{M}$ oleic acid (OA, Sigma- Aldrich, St. Louis, MO) or BSA (control) with or without $3 \mu \mathrm{M}$ PJ34 for $48 \mathrm{~h}$.

\section{Western blotting}

Protein samples (60 $\mu \mathrm{g}$, as determined by a Bio-Rad assay, Thermo, Rockford, IL, USA) were separated by $9 \%$ sodium dodecyl sulfate-polyacrylamide gel electrophoresis. Anti-poly(ADP-ribose) polymer (PAR, Trevigen, Gaithersburg, MD), SREBP1 (SANTA CRUZ Biotechnology, CA) and anti-Actin antibodies were incubated overnight at $4^{\circ} \mathrm{C}$. Actin was used as a control to confirm equal loading of proteins.

\section{Quantitative real-time PCR}

Total RNA was extracted from liver tissue or cells using Trizol (Invitrogen, Frederick, MD, USA), and cDNA was synthesized from $2 \mu \mathrm{g}$ of total RNA using M-MLV reverse transcriptase (Invitrogen). Real-time PCR was performed using a SYBR Green Master Mix Kit in a Lightcycler (iQ5, Bio-rad, USA). PCR primers were constructed for Srebp1a, Srebp1c, Srebp2, Chrebp, and Lxro, based on the published nucleotide sequences of mouse gene regions (Table 1). Hprt served as an internal control gene.

\section{Oil Red $O$ staining}

Liver tissues were fixed in $4 \%$ paraformaldehyde and cut into $10-\mu \mathrm{m}$ slices using a cryostat (Leica CM3050S, Germany). The slides were stained with $0.5 \%$ oil red 0 (Sigma-Aldrich) in isopropanol for 30 min, and then rinsed in $60 \%$ isopropanol and distilled water. The slides were sealed with glycerol and photographed.

\section{Immunoprecipitation (IP) Assay}

The cell lysates were incubated with either the anti-Sp 1 antibody or normal rabbit IgG overnight at $4^{\circ} \mathrm{C}$, and then added protein- $\mathrm{A}+\mathrm{G}$ agarose (Beyotime, China) at $4{ }^{\circ} \mathrm{C}$ for 4 hours. The immunoprecipitates were pelleted by centrifugation and washed 4 times with lysis buffer. Then the immunoprecipitated samples were heated in SDS sample buffer and used for SDS-PAGE analysis. Unspecific IgG was used as negative control. 


\begin{tabular}{|c|c|}
\hline Cellular Physiology & Cell Physiol Biochem 2018;49:645-652 \\
\hline and Biochemistrv & \begin{tabular}{l|l} 
DOI: 10.1159/000493028 \\
Published online: 30 August, 2018
\end{tabular} $\begin{array}{l}\text { O2018 The Author(s). Published by S. Karger AG, Basel } \\
\text { www.karger.com/cpp }\end{array}$ \\
\hline
\end{tabular}

$\begin{array}{lllllllll}C & h & r & o & m & a & t & i & n\end{array}$

Immunoprecipitation

(ChIP) Assay

ChIP experiments were

performed as the instruction manual of Chip assay kit

Table 1. Sequences of primers used for real-time PCR

(Beyotime, China). Hep1-6 cells

\begin{tabular}{lcc}
\hline Gene name & Forward primer & Reverse primer \\
\hline Srebp1a & TAGTCCGAAGCGGGTGGGCGCGGGGCCAT & GATGTCGTTCAAAACGCTGTGTGTCCAGTTC \\
Srebp1c & GTTACTCGAGCCTGCCTTCAGG & CAAGCTTTGGACCTGGGTGTG \\
Srebp2 & GCGTTCTGGAGACCATGGA & ACAAAGTTGCTCTGAAAACAAATC \\
Chrebp & GCATCCTCATCCGACCTTTA & GATGCTTGTGGAAGTGCTGA \\
Lxro & AGGAGTGTCGACTTCGCAAA & CTCTTCTTGCCGCTTCAGTTT \\
\hline
\end{tabular}

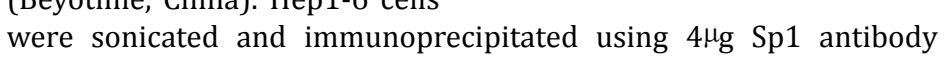
(Abcam, Cambridge, MA) or IgG. The sheared DNA was purified with a DNA extraction kit and amplified by quantitative real-time PCR analysis. The primer sequences of srebf1 promoter were $5^{\prime}$-CCA TCC CTG GCC CTT TAA TCT AAC GA-3' (sense) and 5'-TTC GGA CTA GGC CCA CGT TAA GGA AA-3' (anti-sense).

Table 2. Composition of experimental diets

\begin{tabular}{lcc}
\hline Component & Normal diet & High-fat diet \\
\hline Carbohydrate (\%) & 65.42 & 20 \\
Protein (\%) & 22.47 & 20 \\
Fat $(\%)$ & 12.11 & 60 \\
Vitamins & + & + \\
\hline
\end{tabular}

\section{Animals and diet}

Male C57BL/6 mice (six to eight weeks old) were fed with a normal fat diet or a high fat diet (D12492, Huafukang, China) ad libitum for 3 months (Table 2). Their body weights were monitored weekly. After 3 months, the mice were divided into four groups (six mice per group): (1) normal fat diet mice; (2) normal fat diet mice receiving intraperitoneal injections of $30 \mathrm{mg} / \mathrm{kg}$ doses of PJ34 (Sigma-Aldrich, St. Louis, MO, USA) once a day for two weeks; (3) high fat diet mice; (4) high fat diet mice receiving PJ34 treatment (same as group 2). All these protocols were reviewed and approved by the local animal ethical committee of Beijing normal university.

\section{Intraperitoneal glucose tolerance test (IpGTT)}

At the end of 3 months of high fat feeding, all the mice underwent a glucose tolerance test; and after PJ34 treatment for two weeks, the glucose tolerance test was repeated. The mice were fasted overnight and then received an intraperitoneal injection of $2 \mathrm{~g}$ glucose $/ \mathrm{kg}$ body weight. The procedure was same as that described previously [7].

\section{Intraperitoneal insulin tolerance test (IpITT)}

Following $4 \mathrm{~h}$ of fasting, mice were given an intraperitoneal injection of $0.5 \mathrm{U}$ insulin $/ \mathrm{kg}$ body weight. Glucose was measured from blood samples taken from the tip of the tail at intervals of 15, 30, 60, and 120 min. Mice received an insulin tolerance test both before and after PJ34 administration.

\section{Blood samples and tissue collection}

Mice were fasted overnight and anesthetized using pentobarbital sodium. After euthanasia, whole blood was collected by puncturing the right ventricle of the heart. Serum was isolated by centrifugation and stored at $-80{ }^{\circ} \mathrm{C}$. The tissues were removed, weighed, and stored at $-80{ }^{\circ} \mathrm{C}$. Triglycerides (TG), total cholesterol (TC), high-density lipoprotein cholesterol (HDL), and low-density lipoprotein cholesterol (LDL) were measured using an automatic analyzer (Hitachi, 7600, 7170A).

\section{Insulin and insulin sensitivity indices}

Serum insulin was assayed using a commercial enzyme-linked immunosorbent assay (ELISA) kit (Alpco Diagnostics, Salem, NH, USA), according to the manufacturer's instructions. Insulin resistance was assessed by several indices [8]. The formulae used are shown below:

HOMA-IR $=(\mathrm{FBI}(\mu \mathrm{U} / \mathrm{ml}) \times \mathrm{FBG}(\mathrm{mmol} / \mathrm{L})) / 22.5$

QUICKI $=1 /(\log (\mathrm{FBG} \mathrm{mg} / \mathrm{dL})+\log (\mathrm{FBI} \mu \mathrm{U} / \mathrm{ml}))$

HOMA-IR: Homeostatic model assessment-insulin resistance; QUICKI: quantitative insulin sensitivity check index; FBI: fasting blood insulin; FBG: fasting blood glucose.

\section{Statistical analysis}

Data are expressed as the mean \pm the standard deviation (SD) or the mean \pm the standard error of the mean (SEM). Comparisons between two mean values were performed using an independent samples t-test. For multiple comparisons among different groups of data, significant differences were determined using one-way ANOVA in the SPSS software. To test for differences across time, a one way repeated measures ANOVA with post-hoc tests was used. Differences between values were considered significant at $\mathrm{P}<0.05$. 


\section{Results}

PARP inhibitor PJ34 increased lipid synthesis in HEP1-6 cells

After 2-d oleic acid treatment, the lipid accumulation was significantly enhanced in HEP1-6 cells. When PJ34 was supplemented, the effect of oleic acid upon lipid synthesis was strengthened (Fig. 1A). The total level of poly(ADP-ribosyl)ated protein is expressed as PARP activity. Oleic acid treatment induced PARP activation, whereas PJ34 treatment inhibited PARP activation (Fig. 1B). Compared with oleic acid-treated cells, the expression of srebf1, a lipid synthesis gene, at the mRNA and protein levels was significantly up-regulated in the oleic acid + PJ34-treated cells $(\mathrm{P}<0.05$, Fig. $1 \mathrm{~B}$ and C). To unravel the possible molecular mechanisms, the expression of specificity protein 1 (Sp1), which has been proven to modulate srebf1 expression, was quantitatively measured $[9,10]$. We found that transcription factor $\mathrm{Sp} 1$ was modified by poly(ADPribosyl)ation, although the level of $\mathrm{Sp} 1$ protein was not altered after PJ34 treatment (Fig. 1B and D). PJ34 treatment enhanced the binding of Sp1 to DNA in the SREBF1 promoter, suggesting that PARP inhibition promoted the transcriptional activation of Sp1 (Fig. 1E).

\section{Effects of PJ34 on serum lipid contents \\ Compared with mice}

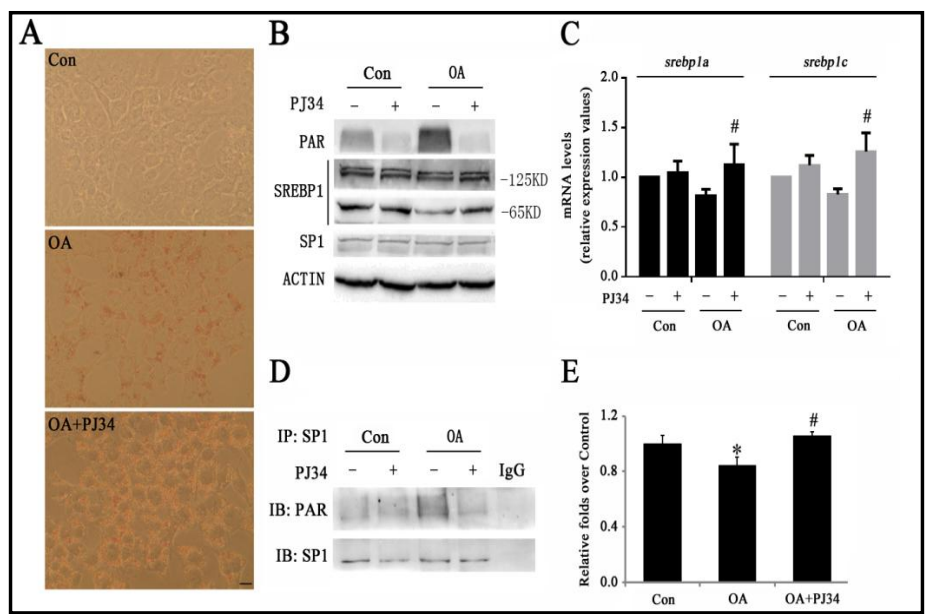

Fig. 1. Effects of PARP inhibition on lipid synthesis in HEP1-6 cells. Lipid accumulation (A), the mRNA levels (C) and protein levels (B) of SREBP1 were detected in HEP1-6 cells treated with oleic acid (OA) with or without PJ34 for 2 days. The modification of SP1 by poly(ADPribosyl)ation was examined by co-IP experiment (D). The recruitment of Sp1 to srebf1 promoter was examined by ChIP assay (E). Actin was used as control to confirm equal loading of proteins. Data are presented as means $\pm S D(n=3)$. $\# P<0.05$ versus $O A$ without $P J 34$. The length of the scale is $20 \mu \mathrm{m}$.

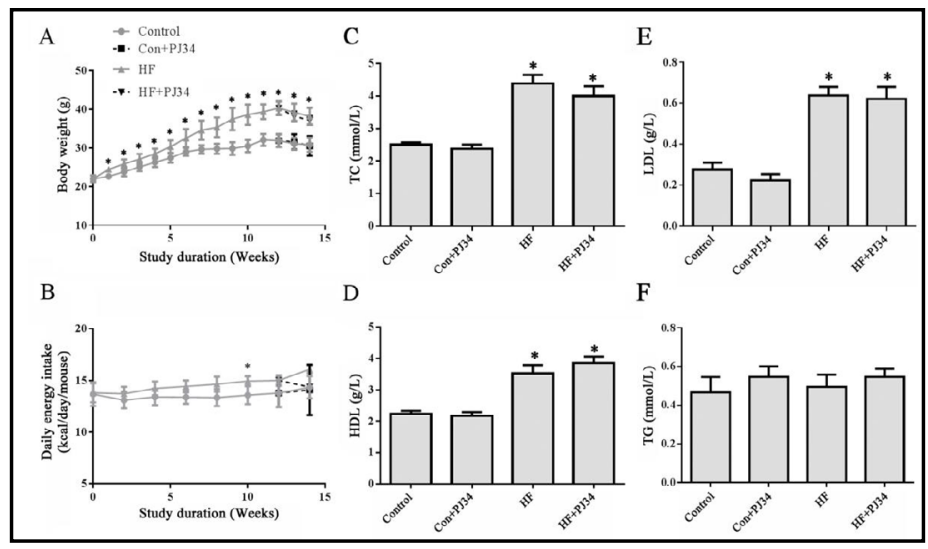

Fig. 2. Effects of PARP inhibition on body weight, energy intake and serum lipid contents. Mice were fed with high fat diet for 12 weeks, and then received $30 \mathrm{mg} / \mathrm{kg}$ PJ34 every day for 2 weeks. Body weight was detected every week (A) and energy intake was measured every other week (B). Serum TC, LDL, HDL and TG concentrations were examined (C-F). Data are presented as means \pm SD $(n=6) .{ }^{*} \mathrm{P}<0.05$ versus control.

fed with a normal-fat diet, mice fed with a high-fat diet for 3 months obtained a significant increase in body weight and calorie intake ( $\mathrm{P}<0.05$, Fig. $2 \mathrm{~A}$ and $2 \mathrm{~B})$. The serum levels of lipid were determined following 3-month feeding. Specifically, the serum levels of TC, LDL and HDL were significantly increased in mice fed with a high-fat diet $(\mathrm{P}<0.05$, Fig. 2C-2E). Nevertheless, subsequent administration of PJ34 did not significantly change the serum levels of these parameters (Fig. 2). The serum level of TG did not significantly change in mice fed with a high-fat diet with or without PJ34 administration (Fig. 2E). 


\section{Cellular Physiology Cell Physiol Biochem 2018;49:645-652 and Biochemistry \begin{tabular}{l|l} 
DOI: 10.1159/000493028 & (c) 2018 The Author(s). Published by S. Karger AG, Basel \\
www.karger.com/cpb
\end{tabular}

Effects of PJ34 on glucose metabolism

The levels of blood glucose and serum insulin were increased markedly in the mice fed with a high-fat diet compared with the control mice $(\mathrm{P}<$ 0.05 , Fig. $3 A$ and $B$ ). Using fasting glucose and insulin concentration to calculate insulin sensitivity indices, both HOMOIR and QUICKI demonstrated that insulin sensitivity was impaired in mice fed with a high-fat diet $(\mathrm{P}<0.05$, Fig. $3 \mathrm{C}$ and $\mathrm{D})$. Treatment with a high-fat diet + PJ34 slightly decreased the blood glucose and insulin concentration with no statistical significance (Fig. 3).

In the IpITT experiment, mice fed with a high-fat diet and treated with PJ34 obtained a significant improvement in the insulin tolerance compared with the mice fed with a high-fat diet alone $(\mathrm{P}<0.05$, Fig. 3F). Nevertheless, the IpGTT experiment revealed no significant change in the glucose tolerance between the two groups (Fig. 3E). In addition, insulin sensitivity was also statistically compared in mice before and after PJ34 treatment. The insulin tolerance was markedly improved after PJ34 treatment in the high-fat dietfed mice $(\mathrm{P}<0.05$, Fig. $3 \mathrm{H})$, whereas no significant effect of PJ34 treatment upon the glucose tolerance was noted (Fig. 3G). PJ34 administration did not induce any change in the insulin sensitivity in the mice fed with a normal-fat diet (Fig. 3).

Effects of PJ34 on lipid synthesis and insulin sensitivity in the liver

As illustrated in Fig. 4A, PARP activity in the mice fed with a high-fat diet was significantly higher compared with that in the control mice. PJ34 administration significantly suppressed the PARP activity $(\mathrm{P}<0.05)$. Phosphorylation of insulin signaling proteins (insulin receptor, AKT and GSK) was markedly down-regulated in mice fed with a high-fat diet and reversed by PJ34 administration $(\mathrm{P}<0.05$, Fig. 4B). The expression level of Srebp1c and Chrebp mRNA, as the lipid synthesis genes, were significantly up-regulated in the liver tissues of mice fed with a high-fat diet + PJ34 compared with those in mice fed with a high-fat diet alone $(\mathrm{P}<0.05$, Fig. $4 \mathrm{C})$. In agreement with this finding, there were more lipid droplets in the liver tissues of mice fed a high-fat diet + PJ34 than mice fed a high-fat diet alone (Fig. 4D). The high-fat diet significantly induced lipid accumulation in the liver, which was subsequently aggravated by PJ34 treatment.

\section{Discussion}

PARP, a classic DNA repair enzyme, may participate in multiple cellular functions by modulating multiple target proteins via the modification of poly(ADP-ribosyl)ation. In this study, short-term PARP inhibition promoted the lipid accumulation and up-regulated 


\section{Cellular Physiology and Biochemistry Published online:30 August, 2018 \begin{tabular}{l|l} 
D 2018 The Author(s). Published by S. Karger AG, Basel \\
www.karger.com/cpb
\end{tabular} \\ Pang et al.: PARP Inhibition Increased Lipid Accumulation}

the expression levels of lipid synthesis genes in mice fed with high-fat diet. The transcription factor, Sp1 may be involved in this process when modified by poly(ADP-ribosyl)ation.

PARP activation represents two seemingly opposing properties. Specifically, PARP activation repairs damaged DNA, and affects cellular energy through NAD depletion [11]. PARP deletion probably exerts both positive and negative effects. A previous study demonstrated that PARP1 deficiency promotes the lipid accumulation in liver [12] and exacerbates high-fat diet-induced obesity [6]. In the present study, shortterm PARP inhibition also enhanced the lipid synthesis in the liver tissues of mice fed with a high-fat diet, indicating that PARP is involved in the lipid metabolism via gene modulation.

Lipid synthesis genes were detected in hepatocytes according to the previous studies [13]. PARP inhibitor treatment up-regulated the expression level of SREBP1 both in vitro and in vivo. Highfree fatty acid-induced poly(ADP-ribosyl) ation modification of Sp1 in Hep1-6 cells results in a lower binding affinity of Sp1 to the promoter regions of SREBP1. Some researchers have demonstrated that PARP1 activation promotes cell cycle progression by inhibiting the Sp1 signaling pathway in hepatoma cells [14]. Consistent with these findings, we observe that PARP is involved in lipid metabolism by modulating Sp1-mediated

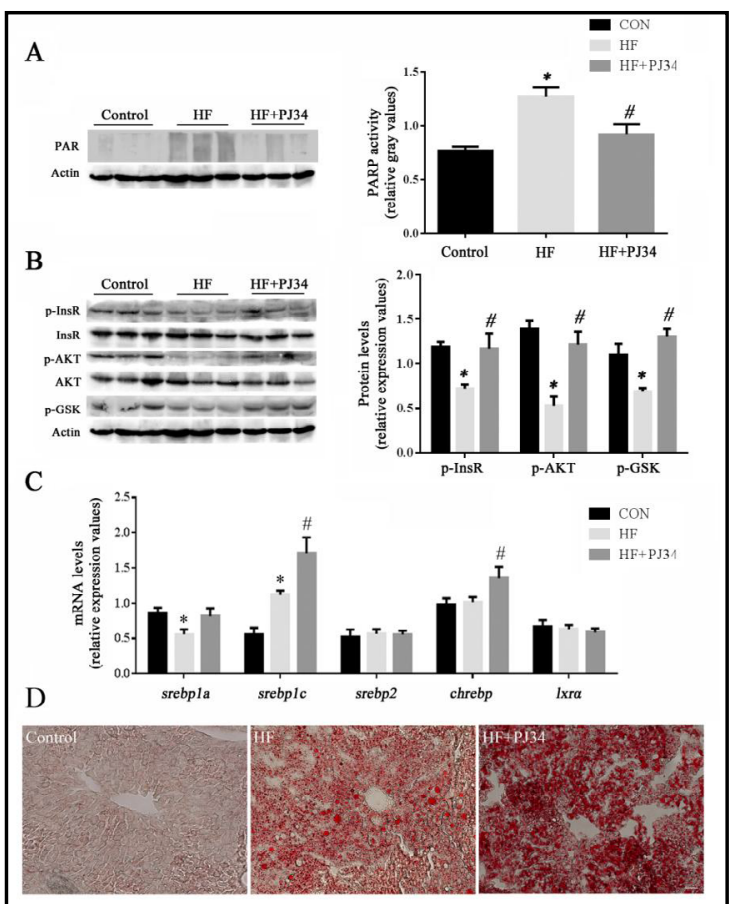

Fig. 4. Effects of PJ34 on PARP activity, lipid synthesis genes and lipid accumulation in the liver. PARP activity (PAR) was detected by western blot (A). The phosphorylation levels of insulin signaling proteins (p-InsR, p-AKT, p-GSK) were detected in the liver tissue (B). The mRNA levels of lipid synthesis genes were examined by real-time PCR (C). And liver tissue slides were stained with oil red $\mathrm{O}$ and photographed under a microscope (D). Data are presented as means \pm SEM $(\mathrm{n}=6) .{ }^{*} \mathrm{P}<0.05$ versus control. $\# \mathrm{P}<0.05$ versus HF. The length of the scale is $20 \mu \mathrm{m}$. SREBP1 expression in hepatocytes.

Interestingly, short-term PARP inhibition slightly increased insulin sensitivity, which contributed to an improvement in glucose metabolism. After PJ34 administration, the insulin signaling pathway in the liver was reversed, insulin tolerance was improved and the levels of blood glucose and insulin were slightly decreased, which were consistent with a previous report in which PARP1-deficient mice fed with a high-fat diet possessed increased insulin sensitivity than wild-type mice fed with a high-fat diet [5]. Previously, we also reported that PJ34 increases the insulin sensitivity by up-regulating intracellular NAD+ levels and SIRT1 activity in hepatocytes [15-17]. According to these observations, PARP inhibition exerts beneficial effects upon the glucose metabolism.

Lipid metabolic dysfunction is always accompanied by glucose metabolic disorders in patients with metabolic syndrome. In the current study, we observed a paradoxical phenomenon; specifically, PJ34 treatment induced the lipid accumulation and improved the insulin sensitivity. PARP can catalyze multiple target proteins in vivo [18]. Our results suggest that different proteins involved in glucose and lipid metabolism may be modulated by PARP simultaneously. During short-term PJ34 treatment, some direct reactions of PARP catalysis were observed, while after long-term observations, some complex interactive effects appeared. Prolonged PJ34 treatment time might have a different effect on lipid and glucose metabolism, which warrants further investigation. In addition, we only observed a molecular change in PARP inhibition in liver tissues. Alterations in other insulin-sensitive tissues which contribute to metabolism also needs to be researched further. 


\section{Cellular Physiology Cell Physiol Biochem 2018;49:645-652 \begin{tabular}{ll|l} 
and Biochemistry Published online: 30 August, 2018 & $\begin{array}{l}\text { (c) } 2018 \text { The Author(s). Published by S. Karger AG, Basel } \\
\text { www.karger.com/cpb }\end{array}$
\end{tabular}}

Pang et al.: PARP Inhibition Increased Lipid Accumulation

\section{Conclusion}

Short-term PARP inhibitor administration increases the expression of fatty acid synthesis genes and induced liver lipid accumulation; however, it also improved high-fat diet-induced insulin resistance. Accordingly, we hypothesized that PARP plays an important role in metabolism through multiple target genes post-modification, which requires further research. The therapeutic application of PARP inhibitors in metabolism should be seriously considered in the future.

\section{Acknowledgements}

This work was supported by National Natural Science Foundation of China (No: 81100570 and 31400995), 12th five-year National Science and Technology Project (No: 2012BAI10B01) and Beijing Natural Science Foundation (No: 7154234).

\section{Disclosure Statement}

The authors have no conflict of interests to disclose.

\section{References}

1 Cantó C, Sauve AA, Bai P: Crosstalk between poly(ADP-ribose) polymerase and sirtuin enzymes. Mol Aspects Med 2013;34:1168-1201.

2 D'Amours D, Desnoyers S, D'Silva I, Poirier GG: Poly(ADP-ribosyl)ation reactions in the regulation of nuclear functions. Biochem J 1999;342:249-268.

3 Rösen P, Nawroth PP, King G, Möller W, Tritschler HJ, Packer L: The role of oxidative stress in the onset and progression of diabetes and its complications: A summary of a Congress Series sponsored by UNESCOMCBN, the American Diabetes Association and the German Diabetes Society. Diabetes Metab Res Rev 2001;17:189-212.

4 Yang H, Jin X, Kei Lam CW, Yan SK: Oxidative stress and diabetes mellitus. Clin Chem Lab Med 2011;49:1773-1782.

5 Bai P, Canto C, Oudart H, Brunyánszki A, Cen Y, Thomas C, Yamamoto H, Huber A, Kiss B, Houtkooper RH, Schoonjans K, Schreiber V, Sauve AA, Menissier-de Murcia J, Auwerx J: PARP-1 inhibition increases mitochondrial metabolism through SIRT1 activation. Cell Metab 2011;13:461-468.

-6 Devalaraja-Narashimha K, Padanilam BJ: PARP1 deficiency exacerbates diet-induced obesity in mice. J Endocrinol 2010;205:243-252.

7 Pang J, Xi C, Huang XQ, Cui J, Gong H, Zhang TM: Effects of excess energy intake on glucose and lipid metabolism in C57BL/6 mice. PLoS One 2016;11:e0146675.

$>8$ Jaya C, Anuradha CV: Cissus quadrangularis stem alleviates insulin resistance, oxidative injury and fatty liver disease in rats fed high fat plus fructose diet. Food Chem Toxicol 2010;48:2021-2029.

-9 Penque BA, Hoggatt AM, Herring BP, Elmendorf JS: Hexosamine biosynthesis impairs insulin action via a cholesterolgenic response. Mol Endocrinol 2013;27:536-547.

-10 Solomon SS, Majumdar G, Martinez-Hernandez A, Raghow R: A critical role of Sp1 transcription factor in regulating gene expression in response to insulin and other hormones. Life Sci 2008;83:305-312.

11 Virág L, Szabó C: The therapeutic potential of poly(ADP-ribose) polymerase inhibitors. Pharmacol Rev 2002;54:375-429.

-12 Erener S, Mirsaidi A, Hesse M, Tiaden A, Ellingsgaard H, Kostadinova R, Donath MY, Richards PJ, Hottiger MO: ARTD1 deletion causes increased hepatic lipid accumulation in mice fed a high-fat diet and impairs adipocyte function and differentiation. FASEB J 2012;26:2631-2638.

13 Wang L, Zhang N, Wang Z, Ai DM, Cao ZY, Pan HP: Decreased MiR-155 level in the peripheral blood of nonalcoholic fatty liver disease patients may serve as a biomarker and may influence LXR activity. Cell Physiol Biochem 2016;39:2239-2248.

14 Yang L, Huang K, Li X, Du M, Kang X, Luo X, Gao L, Wang C, Zhang Y, Zhang C, Tong Q Huang K, Zhang F, Huang D: Identification of poly(ADP-ribose) polymerase-1 as a cell cycle regulator through modulating Sp1 mediated transcription in human hepatoma cells. PLoS One 2013;8:e82872.

15 Pang J, Cui J, Gong H, Xi Chao, Zhang TM: Effects of NAD on PARP-mediated insulin sensitivity in oleic acid treated hepatocytes. J Cell Physiol 2015;230:1607-1613. 


\section{Cellular Physiology Cell Physiol Biochem 2018;49:645-652 \begin{tabular}{l|l|l} 
DOI: 10.1159/000493028 & $\begin{array}{l}\text { O 2018 The Author(s). Published by S. Karger AG, Basel } \\
\text { www.karger.com/cpb }\end{array}$
\end{tabular}

Pang et al.: PARP Inhibition Increased Lipid Accumulation

16 Pang J, Gong H, Xi C, Fan W, Dai Y, Zhang TM: Poly(ADP-Ribose) polymerase 1 is involved in Glucose toxicity through SIRT1 modulation in HepG2 hepatocytes. J Cell Biochem 2011;112:299-306.

17 Pang J, Xi C, Jin JH, Han YW, Zhang TM: Relative quantitative comparison between lipotoxicity and glucotoxicity affecting the PARP-NAD-SIRT1 pathway in hepatocytes. Cell Physiol Biochem 2013;32:719727.

18 D’Amours D, Desnoyers S, D’Silva I, Poirier GG: Poly(ADP-ribosyl)ation reactions in the regulation of nuclear functions. Biochem J 1999;342:249-268. 